\title{
Revealing the excitation energy transfer network of Light-Harvesting Complex II by a phenomenological analysis of two-dimensional electronic spectra at $77 \mathrm{~K}$
}

\author{
Cite as: J. Chem. Phys. 151, 205101 (2019); doi: 10.1063/1.5125744 \\ Submitted: 29 August 2019 - Accepted: 5 November 2019 • \\ Published Online: 27 November 2019
}

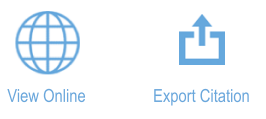

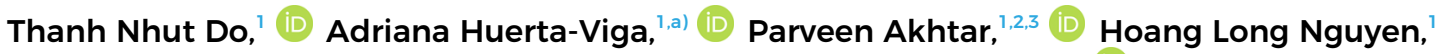

Pawet J. Nowakowski, ${ }^{,}$M. Faisal Khyasudeen, ${ }^{1, b)}$ Petar H. Lambrev, ${ }^{2, c)}$ (D) and Howe-Siang Tan ${ }^{1, c)}$ (D)

\author{
AFFILIATIONS \\ ${ }^{1}$ Division of Chemistry and Biological Chemistry, School of Physical and Mathematical Sciences, Nanyang \\ Technological University, 21 Nanyang Link, Singapore 637371 \\ ${ }^{2}$ Biological Research Centre, Szeged, Temesvári Körút 62, Szeged 6726, Hungary \\ ${ }^{3}$ ELI-ALPS, ELI-HU Nonprofit Ltd., Budapesti út 5, Szeged, Hungary
}

Note: The paper is part of the JCP Special Topic on Ultrafast Molecular Sciences by Femtosecond Photons and Electrons.

a) Current address: Institute of Biophysics, Johann Wolfgang Goethe-University, Max-von-Laue-Str. 1, 60438

Frankfurt am Main, Germany.

b) Current address: Department of Chemistry, Faculty of Science, University of Malaya, 50603 Kuala Lumpur, Malaysia.

${ }^{c)}$ Authors to whom correspondence should be addressed: lambrev.petar@brc.hu and howesiang@ntu.edu.sg

\begin{abstract}
Energy equilibration in light-harvesting antenna systems normally occurs before energy is transferred to a reaction center. The equilibration mechanism is a characteristic of the excitation energy transfer (EET) network of the antenna. Characterizing this network is crucial in understanding the first step of photosynthesis. We present our phenomenology-based analysis procedure and results in obtaining the excitonic energy levels, spectral linewidths, and transfer-rate matrix of Light-Harvesting Complex II directly from its 2D electronic spectra recorded at $77 \mathrm{~K}$ with waiting times between $100 \mathrm{fs}$ to $100 \mathrm{ps}$. Due to the restriction of the models and complexity of the system, a unique EET network cannot be constructed. Nevertheless, a recurring pattern of energy transfer with very similar overall time scales between spectral components (excitons) is consistently obtained. The models identify a "bottleneck" state in the 664-668 nm region although with a relatively shorter lifetime ( $\sim 4-6 \mathrm{ps}$ ) of this state compared to previous studies. The model also determines three terminal exciton states at $675,677-678$, and 680-681 nm that are weakly coupled to each other. The excitation energy equilibration between the three termini is found to be independent of the initial excitation conditions, which is a crucial design for the light-harvesting complexes to ensure the energy flow under different light conditions and avoid excitation trapping. We proposed two EET schemes with tentative pigment assignments based on the interpretation of the modeling results together with previous structure-based calculations and spectroscopic observables.
\end{abstract}

Published under license by AIP Publishing. https://doi.org/10.1063/1.5125744

\section{INTRODUCTION}

During the first step of photosynthesis, incident photons from sunlight are harvested by light-sensitive pigment-protein antenna complexes, where the excitation energy is equilibrated before being transferred to the photosynthetic reaction center. The arrangement of antenna complexes around a reaction center is such that nearly every photon absorbed drives photochemistry. ${ }^{1}$ LightHarvesting Complex II (LHCII), the peripheral antenna of Photosystem II (PSII), is the most abundant antenna complex in the 
biosphere. In nature, LHCII mostly exists in a trimeric form, in which each monomeric unit is comprised of eight chlorophylls (Chls) $a$, six Chls $b$, and four xanthophylls. ${ }^{2}$ These pigments are noncovalently bound within the three transmembrane $a$-helices. The protein environment of LHCII optimizes the arrangement of the pigment molecules that enables the ultrafast excitation energy transfer (EET) that normally occurs in femtosecond to picosecond time scales.

The dynamic of the EET process in LHCII has been studied intensively using various spectroscopic techniques. ${ }^{3}$ The general conclusions from these studies are that EET from Chl $b$ to Chl $a$ takes place rapidly, with subpicosecond characteristic times in the range of $0.15-0.6 \mathrm{ps}$ at room temperature and $0.3-0.6 \mathrm{ps}$ at low temperature. ${ }^{4-11}$ This fast phase is followed by slower (several ps) transfers from Chls absorbing in an intermediate region (660$670 \mathrm{~nm}$ ) between the major $\mathrm{Chl} b$ and Chl $a Q_{y}$ bands. The information about the electronic energy level structure of the excited states, which is largely contributed by interactions between Chls and Chlsprotein, is key to understanding the ultrafast dynamics of the EET network.

With the determination of the high-resolution crystal structure of trimeric LHCII, ${ }^{2,12}$ several theoretical studies have been performed in an effort to establish a comprehensive picture of the EET network in LHCII. The proposed models employ various levels of theory such as Förster energy transfer, ${ }^{13}$ Redfield and modified Redfield, ${ }^{14,15}$ and combined modified Redfield-generalized Förster. ${ }^{16,17}$ The most advanced models proposed by Novoderezhkin et al. ${ }^{16}$ and Renger et al. ${ }^{17}$ use modified Redfield-generalized Förster theorygrouping the Chls in the complex into a few strongly coupled clusters. EET within each cluster is treated with modified Redfield theory and between different clusters with the generalized Förster formalism. These two models have achieved consistent results with the experimental observations mentioned above. While there are similarities between these two advanced models, there are also important differences between them. In the Redfield-Förster picture proposed by Novoderezhkin et al., ${ }^{16}$ the weak excitonic coupling between any vibronic transitions of the pigments plays an important role and results in exciton delocalization. The model of Renger et al. ${ }^{17}$ assumes that the protein vibrations can destroy any coherence and lead to exciton localization. As a result of these different approaches, the EET rates obtained by Novoderezhkin et al. are relatively faster.

In recent years, two-dimensional electronic spectroscopy (2DES) has been employed to characterize ultrafast processes in photosynthetic systems. ${ }^{18}$ With one more spectral dimension compared to transient absorption (TA) spectroscopy, 2DES enables the detection of spectrally resolved features of the donor-acceptor pairs participating in the EET processes. Several 2DES studies have been conducted on LHCII at both physiological ${ }^{19-22}$ and cryogenic temperatures. $^{23-25}$ At physiological conditions, the 2DES study conducted by Wells et al. revealed different transfer pathways between the high and low energy Chls $b$ in LHCII. ${ }^{19}$ The EET from high energy Chl $b$ directly to $\mathrm{Chl} a$ was detected with a subpicosecond rate. The low energy Chls $b$ shows multistep dynamics with subpicoseconds to several picoseconds transfers to intermediate states followed by few-picoseconds transfers to the final Chl $a$. The equilibration between the Chls a manifold was resolved by Akhtar et al. and found to occur within a few picoseconds. ${ }^{22}$ At $77 \mathrm{~K}$, the 2DES measurement of LHCII conducted by Schlau-Cohen et al. reported sub-100 fs relaxation rates between the strongly coupled Chls and a few to tens of picoseconds rates of intercluster transfer processes. $^{25}$ By observing quantum beats in nonrephasing $2 \mathrm{D}$ electronic spectra, Calhoun et al. could determine 14 excitonic energy levels in LHCII at $77 \mathrm{~K}^{23}$ More recently, 2DES has been applied to probe the energy transfer in LHCII in a range of temperatures from $77 \mathrm{~K}$ up to room temperature ${ }^{26}$ with substantial improvement in the resolution of spectral components at $77 \mathrm{~K}$. Although valuable spectroscopic information has been unveiled with $2 \mathrm{DES}$, a complete characterization of the EET network of LHCII with one to one mapping between the excitonic levels and pigment identities remains elusive. In this study, we report on our approach to extract physical parameters and mechanistic information about the exciton states, EET pathways, and dynamics from 2DES measurements by phenomenological model fitting. This approach was applied to model the 2DES data of trimeric LHCII obtained at $77 \mathrm{~K}$ and propose tentative EET schemes that compare well with the modified Redfield-generalized Förster models mentioned above. Without a substantial a priori knowledge about the complex, the phenomenological approach can construct reasonable EET schemes with the determination of three lowest energy sinks absorbing at $675,677-678$, and $680-681 \mathrm{~nm}$ with no significant EET connecting them. The energy equilibration between these three lowest energy states occurs via uphill energy flow to higher states, which is partially the reason for the observed temperature dependence of the energy equilibration process. ${ }^{26}$ Although the complete EET scheme cannot be determined uniquely from the available experimental data, the phenomenological approach can provide a simple but potentially powerful tool to investigate the EET network in photosynthetic complexes, whose atomistic structures have not been resolved.

\section{METHODOLOGY}

Here, we present the procedure to obtain a phenomenological description of the EET network in LHCII from 2DES data. The experimental details about our annihilation-free 2DES measurements are comprehensively described in a separated article. ${ }^{26}$ Briefly, a pair of excitation pulses with controllable interpulse delay time and phase was obtained by passing the output of a noncollinear parametric amplifier (TOPAS White, Light Conversion) through an acousto-optic programmable pulseshaper (Dazzler, Fastlite). The excitation pulse spectrum was centered at $675 \mathrm{~nm}$, coinciding with the $\mathrm{Q}_{\mathrm{y}}$ absorption band of Chl $a$. After a waiting time $T_{w}$, a white light pulse generated from a 2-mm sapphire window probes the excited state and generates the third order polarization, which is then heterodyne-detected on a spectrometer. The delay $\tau$ between two excitation pulses is scanned to retrieve the coherence occurring between two excitations. The signals were then Fourier transformed along $\tau$ to yield the final 2D electronic spectra.

The principles of the modeling method were proposed by Dostál et al. ${ }^{27}$ and were implemented to analyze the EET network of Fenna-Matthews-Olson (FMO) complex of green sulfur bacteria by Thyrhaug et al. ${ }^{28}$ In that work, the fitting procedure is conducted to extract the excitonic energy levels, spectral lineshape of each exciton, and the transfer-rate matrix based on the $2 \mathrm{D}$ electronic 
spectra. However, spectral features of the FMO complex are well separated and individual exciton states are identifiable in the 2D spectra. In contrast, LHCII exhibits highly congested 2D electronic spectra; hence, the criterium of good discernibility of spectral peaks ${ }^{27}$ is not satisfied and the modeling approach needs to be modified to resolve the EET network.

The parameters of the model are excitonic energy levels, the spectral linewidth of each exciton, the transfer-rate matrix containing information about the EET between excitons, a matrix containing information of the cross-peaks at zero waiting time (for example, arising due to exciton coupling), and a phenomenological excited-state absorption (ESA) spectrum. A nonlinear least-square fitting routine is implemented to fit the modeled $2 \mathrm{D}$ electronic spectra to the experimental data. In this way, we can obtain valuable information about the excitons and energy transfer network in LHCII.

According to the study of Dostál et al., ${ }^{27}$ provided the system meets a set of criteria, the corresponding 2D electronic spectra are proven to be fully described by

$$
\mathbb{S}_{2 \mathrm{D}}\left(\omega_{t}, T_{w}, \omega_{\tau}\right)=L_{3} \operatorname{diag}\left(\boldsymbol{\mu}^{2}\right) \mathbf{F} e^{\mathbf{K} T_{w}} \operatorname{diag}\left(\boldsymbol{\mu}^{2}\right) L_{1}^{T},
$$

where $L_{1}$ and $L_{3}$ are the matrices containing information about the lineshape along $\omega_{\tau}$ and $\omega_{t}$ (excitation and detection) dimensions, respectively, $\operatorname{diag}\left(\boldsymbol{\mu}^{2}\right)$ is the diagonal matrix with elements equal to the squared excitonic transition dipole moments, $\mathbf{K}$ is the transferrate matrix with an explicit form presented later, and the matrix $\mathbf{F}$ defines the species-associated spectra in a way that the elements $\mu_{i}^{2} F_{i j}$ characterize the net TA signal detected at $\omega_{t}=\omega_{0 \rightarrow i}$ caused by the unit population present at the $j$ th exciton. Dostál et al. ${ }^{27}$ present the explicit form of matrix $\mathbf{F}$ as

$$
\mathbf{F} \propto \mathbb{I}+\mathbf{U}+\mathbf{F}_{\mathrm{ESA}},
$$

where $\mathbb{I}$ is the identity matrix corresponding to the stimulated emission (SE) signal, $\mathbf{U}$ is a matrix containing all elements equal to one representing ground-state bleach (GSB), and $\mathbf{F}_{\mathrm{ESA}}$ is a matrix describing the ESA signal. In this study, due to the small contribution of the ESA compared to the GSB and SE signals, we do not include $\mathbf{F}_{\mathrm{ESA}}$ into the matrix $\mathbf{F}$. We also alter the form of our matrix $\mathbf{F}$ as

$$
\mathbf{F}=\mathbb{I}+\mathbf{X},
$$

where $\mathbb{I}$ is the identity matrix corresponding to the diagonal SE and GSB signals and $\mathbf{X}$ is a matrix with off-diagonal elements only, describing the off-diagonal GSB at zero waiting time.

Using Eq. (1), the previous studies described 2D electronic spectra with the totally uncorrelated lineshape, i.e., the $2 \mathrm{D}$ lineshape is factorizable into the linear spectral lineshape matrix $L_{1}$ and $L_{3}{ }^{27,28}$ In this work, we remove the lineshape factors $L_{1}$ and $L_{3}$ in Eq. (1) and we can obtain an expression of the so-called intensity 2D matrix as

$$
\mathbf{I}^{2 \mathrm{D}}\left(T_{w}\right)=\operatorname{diag}\left(\boldsymbol{\mu}^{2}\right) \mathbf{F} \exp \left(\mathbf{K} T_{w}\right) \operatorname{diag}\left(\boldsymbol{\mu}^{2}\right),
$$

which, for a system of $N$ excitons, is an $N \times N$ matrix describing the intensity of each individual 2D peak in the $2 \mathrm{D}$ electronic spectra at waiting time $T_{w}$. The $2 \mathrm{D}$ lineshape function $S_{i j}^{2 \mathrm{D}}\left(\omega_{t}, \omega_{\tau}\right)$ is described by a bivariance $2 \mathrm{D}$ Gaussian distribution controlled by the linewidths of the $i$ th and $j$ th excitons. A detailed explanation and explicit form of the $2 \mathrm{D}$ peakshape is shown in the supplementary material. Peak amplitude beatings attributed to vibrational or vibronic coherences in $2 \mathrm{D}$ electronic spectra are a common feature observed in several light-harvesting complexes. ${ }^{29-31}$ However, for our experiments, the measured quantum beats are small compared to the energy transfer dynamics and quickly vanish within 300 fs. Hence, we do not take the beating dynamics into consideration in our fit. More details can be found in the supplementary material.

With the expression in Eq. (4) and the 2D lineshape functions of the $N \times N$ spectral peaks, the $2 \mathrm{D}$ electronic spectra of an $\mathrm{N}$-chromophore system can be calculated and a nonlinear least squares fitting routine can be constructed to obtain the kinetic information of the system contained in the transfer-rate matrix $\mathbf{K}$.

The fitting routine is constructed as follows. We describe the Chl pigments in LHCII by $N$ excitonic levels, and the input parameters of the fit take the form

$$
\begin{aligned}
\mathbf{E} & =\left[E_{1}, E_{2}, \ldots, E_{N}\right]^{T}, \\
\boldsymbol{\mu} & =\left[\mu_{1}, \mu_{2}, \ldots, \mu_{N}\right]^{T}, \\
\boldsymbol{\sigma} & =\left[\sigma_{1}, \sigma_{2}, \ldots, \sigma_{N}\right]^{T},
\end{aligned}
$$

where $\mathbf{E}, \boldsymbol{\mu}$, and $\boldsymbol{\sigma}$ are vector arrays with elements $E_{i}, \mu_{i}$, and $\sigma_{i}$ corresponding to the excitonic energy level, the transition dipole moment, and the linear linewidth of the $i$ th exciton, respectively.

In addition, the $\mathbf{X}$ matrix representing the relative cross-peak intensities at zero waiting time [Eq. (6)], the phenomenological ESA spectrum $\mathbf{S}_{\mathrm{ESA}}^{2 \mathrm{D}}\left(\omega_{t}, \omega_{\tau}\right)$, and the transfer-rate matrix $\mathbf{K}$ are also necessary inputs to simulate the $2 \mathrm{D}$ electronic spectra,

$$
\mathbf{X}=\left(\begin{array}{cccc}
0 & X_{12} & \cdots & X_{1 N} \\
X_{21} & 0 & \cdots & X_{2 N} \\
\vdots & \vdots & \ddots & \vdots \\
X_{N 1} & X_{N 2} & \cdots & 0
\end{array}\right) .
$$

The values of elements $X_{i j}$ of the $\mathbf{X}$ matrix are constrained between -0.5 and 0.5 . These cross-peaks originate from the excitonic coupling between pigments creating the off-diagonal GSB signals. Due to the limited time resolution of the experiment and the lack of reliable experimental $2 \mathrm{D}$ spectrum at $T_{w}=0$, crosspeaks arising as a result of very fast exciton relaxation (subhundred femtosecond transfers) can also be indistinguishably modeled by $\mathbf{X}$. In such case, the absolute value of $X_{i j}$ elements can be higher than 0.5 , but the model is not strictly physically realistic. Therefore, we keep the limits of \pm 0.5 for the $X_{i j}$ elements and treat it as an indication for fast exciton relaxations that remains underestimated in the model. Furthermore, because the early-time 2D spectra $\left(T_{w}<300 \mathrm{fs}\right)$ do not show any significant cross-peaks in the uphill region $\left(\lambda_{t}<\lambda_{\tau}\right)$, we only include the lower-half (downhill) region of the $\mathbf{X}$ matrix into the fit, while setting uphill $X_{i j}$ elements to zero.

The transfer-rate matrix $\mathbf{K}$ containing all information about the EET rates takes the form of Eq. (7), where the rate constant $k_{i j}$ is the EET rate constant of the transfer from the $j$ th exciton to the $i$ th exciton, 


$$
\mathbf{K}=\left(\begin{array}{cccc}
-\sum_{i} k_{i 1} & k_{12} & \cdots & k_{1 N} \\
k_{21} & -\sum_{i} k_{i 2} & \cdots & k_{2 N} \\
\vdots & \vdots & \ddots & \vdots \\
k_{N 1} & k_{N 2} & \cdots & -\sum_{i} k_{i N}
\end{array}\right)
$$

The ratio between downhill and uphill EET rates is fixed by the Boltzmann factor $\frac{k_{i j}}{k_{j i}}=\exp \left(\frac{E_{j}-E_{i}}{k_{B} T}\right)$, where $E_{i}$ and $E_{j}$ are the excitonic energies of the $i$ th and $j$ th excitons, $k_{B}$ is the Boltzmann constant, and $T$ is the absolute temperature. This constraint arises from the detailed-balance condition which ensures that the ratio of forward/reverse (uphill/downhill) rate constants is dictated by the Boltzmann distribution function. ${ }^{32}$ The diagonal element of $\mathbf{K}$ is the population relaxation rate of the $i$ th exciton which includes a fixed 2 -ns decay $k_{i i}$ to the ground state.

With the input parameters shown in Eqs. (5) - (7), the intensity $2 \mathrm{D}$ matrix I $\mathrm{I}^{2 \mathrm{D}}\left(T_{w}\right)$ can be calculated using Eq. (4). With the properly calculated $2 \mathrm{D}$ peakshapes, the $2 \mathrm{D}$ electronic spectra $\mathrm{S}^{2 \mathrm{D}}\left(\omega_{t}, T_{w}, \omega_{\tau}\right)$ at any population time $T_{w}$ are simulated as

$$
\mathbf{S}^{2 \mathrm{D}}\left(\omega_{t}, T_{w}, \omega_{\tau}\right)=\sum_{i=1}^{N} \sum_{j=1}^{N} I_{i j}^{2 \mathrm{D}}\left(T_{w}\right) S_{i j}^{2 \mathrm{D}}\left(\omega_{t}, \omega_{\tau}\right)+\mathbf{S}_{\mathrm{ESA}}^{2 \mathrm{D}}\left(\omega_{t}, \omega_{\tau}\right),
$$

where $I_{i j}^{2 \mathrm{D}}\left(T_{w}\right)$ and $S_{i j}^{2 \mathrm{D}}\left(\omega_{t}, \omega_{\tau}\right)$ are elements of the intensity $2 \mathrm{D}$ matrix calculated in Eq. (4) and the corresponding 2D peakshape, respectively. The $\mathbf{S}_{\mathrm{ESA}}^{2 \mathrm{D}}\left(\omega_{t}, \omega_{\tau}\right)$ term is the phenomenological ESA spectrum calculated from the linear absorption, fluorescence, and TA spectra. A detailed description of the phenomenological ESA spectrum can be found in the supplementary material.

So far, we have not considered finite-bandwidth effects of the excitation pulse spectrum. From the experimental EET time scales resolved from global analysis $(0.4,2.7$, and $15 \mathrm{ps})$, the excitation pulse duration of $50 \mathrm{fs}$ is shorter than the observed dynamics. Therefore, the effect of finite bandwidth in $2 \mathrm{D}$ electronic spectra can be incorporated using the approximated expressions derived by Do et $a .^{33}$ as

$$
\mathbb{S}^{2 \mathrm{D}}\left(\omega_{t}, T_{w}, \omega_{\tau}\right)=I_{\mathrm{exc}}\left(\omega_{\tau}\right) \mathbf{S}^{2 \mathrm{D}}\left(\omega_{t}, T_{w}, \omega_{\tau}\right),
$$

where $I_{\text {exc }}\left(\omega_{\tau}\right)$ is the intensity spectrum of the excitation pulse, needed to incorporate finite-pulse effects. ${ }^{33}$ We do not need the spectral dependence of the probe pulse along $\omega_{t}$ (detection wavelength) because the finite-pulse effects of the probe are automatically eliminated with phase cycling (similarly, the differential absorbance in conventional TA measurements is independent of the probe intensity, within a range)

We used a nonlinear least squares optimization procedure minimizing the $\chi^{2}$ statistic to fit the model described above to the 2D electronic spectra having $T_{w}=100$ fs to 100 ps. A series of fits were conducted to determine the number of excitonic levels that are necessary for a reasonable goodness of fit. Next, to determine the uniqueness of the best-fit solution and to ensure reaching a global $\chi^{2}$ minimum, the optimization was repeated 1600 times with random initial values of the model parameters. The fitting results were sorted based on the residuals from the best fit (lowest residual) to the worst fit (highest residual), and the best 120 fits are chosen to be further interpreted.

\section{RESULTS AND DISCUSSION}

\section{A. Excitonic states}

Fitting the experimental 2D electronic spectra with varying numbers of exciton states evidently results in solutions that greatly differ in the goodness-of-fit, evaluated by the $\chi^{2}$ statistic, the amplitude of the residuals, and the qualitative likeness of the fits and experimental data. We determined that a minimum of eight exciton states was necessary for an adequate description of the data. Increasing the number of excitons did improve the fit, however, at the expense of a sharp increase in the correlations between fit parameters, especially in the transfer-rate matrix, and number of solutions, which makes the results difficult to interpret. Therefore, we restrict the analysis to eight states.

A histogram of the exciton levels of the 120 best fits (lowest $\chi^{2}$ ), selected among 1600 eight-exciton fits with random starting parameters, is shown in Fig. 1. The relatively low excitation intensity below $660 \mathrm{~nm}$ (respectively, a lower signal-to-noise level in the data) and the short $\mathrm{Chl} b$ lifetimes preclude accurate modeling of Chl $b$ dynamics. Hence, this region was modeled as a single exciton level with a fixed position at $655 \mathrm{~nm}$ that serves as the representative state for the Chl $b$ manifold. The remaining seven exciton levels were free fit parameters. In Fig. 1, we can see that excitonic states are well-defined with narrow distributions, especially for the five lowest levels with

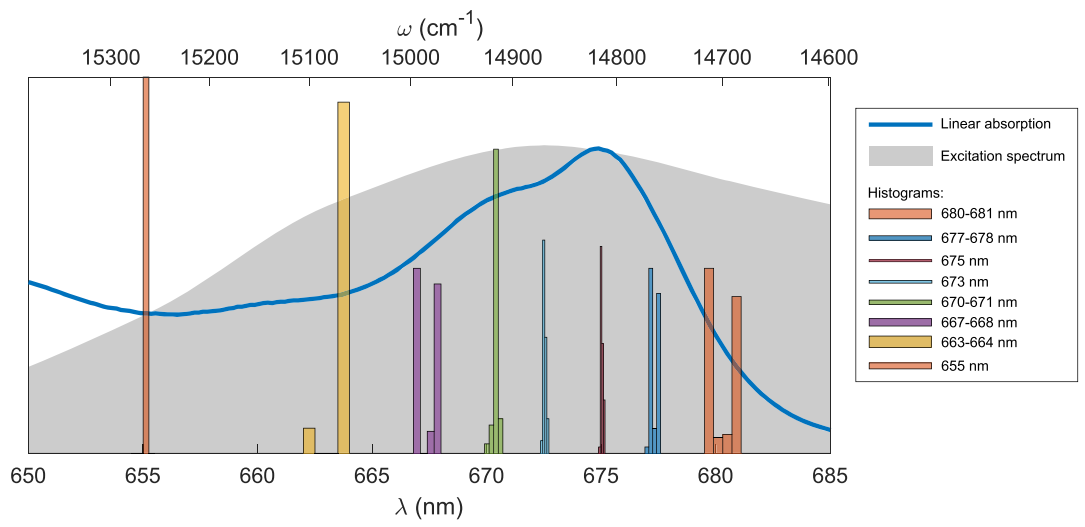

FIG. 1. Distributions of the excitonic energy levels obtained from the best 120 fitting results (histograms) overlaid with the experimental linear absorption spectrum of LHCII at $77 \mathrm{~K}$ (thick blue line) and the laser excitation spectrum used in the 2DES measurements (gray shaded area). The thickness of the bars in each histogram is proportional to the uncertainty of the corresponding excitonic level. 
$655 \mathrm{~nm}$

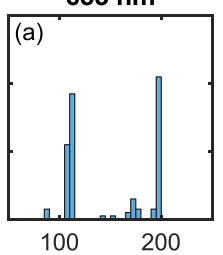

$673 \mathrm{~nm}$

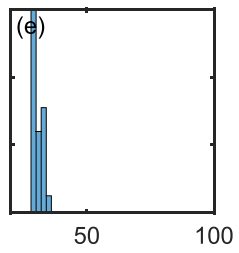

662-664 nm

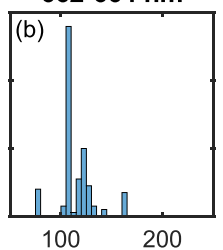

$675 \mathrm{~nm}$

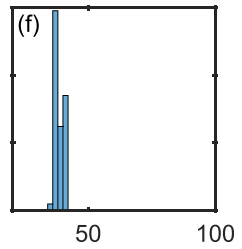

666-668 nm

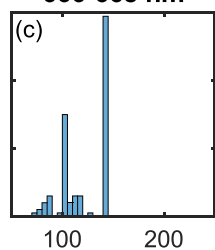

677-678 nm

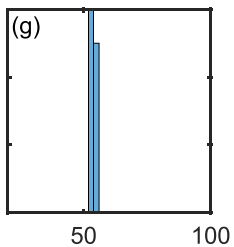

670-671 nm

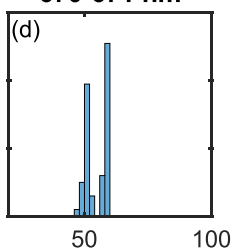

680-681 nm

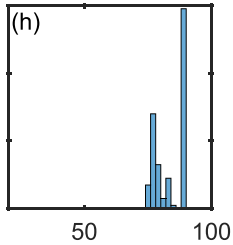

FIG. 2. $[(a)-(h)]$ Inhomogeneous linewidth (half-width at half-maximumHWHM) distributions of the eight excitonic levels obtained from the best 120 fits. The bold title of each panel presents the wavelength of the corresponding excitonic level, and the horizontal axis shows the linewidth in unit of $\mathrm{cm}^{-1}$ the centers at $670-671,673,675,677-678$, and $680-681 \mathrm{~nm}$. The two higher-energy exciton states at 662-664 nm and 666-668 nm are resolved with relatively lower accuracy.

As shown in the supplementary material, Table S1, the lowest five excitonic levels obtained by our model are highly consistent with the determination by Calhoun et al. based on coherence beating with an error margin of $\pm 10 \mathrm{~cm}^{-1} .{ }^{23}$ In structure-based calculations of Novoderezhkin et al., ${ }^{16}$ there is no exciton absorbing at $673 \mathrm{~nm}$. Instead, there are two excitons located at the $670-671 \mathrm{~nm}$ region and two others at the $677-678 \mathrm{~nm}$ region. On the other hand, the electrostatic quantum calculation of Renger et al. ${ }^{17}$ predicts only three absorption levels above $670 \mathrm{~nm}$ : $a 610$ pigment at $679 \mathrm{~nm}, a 602$ and $a 613$ are degenerate at $675 \mathrm{~nm}$, and $a 604$ with $a 603$ absorb at $673 \mathrm{~nm}$.

Figure 2 shows the distributions of inhomogeneous linewidths (half-width at half-maximum-HWHM) of the eight fitted excitonic levels. In the low-energy region $(>670 \mathrm{~nm})$, most states have linewidths ranging between 30 and $55 \mathrm{~cm}^{-1}$ [Figs. 2(d)-2(g)], except for the lowest energy state at $681 \mathrm{~nm}$ having $\sim 80 \mathrm{~cm}^{-1}$ linewidth [Fig. 2(h)]. The broadening of the lowest energy state could be due to several reasons. On the one hand, the lineshapes are modeled as a Gaussian, which could be inaccurate in the real system-the lowenergy tail of the spectrum seems to deviate from a Gaussian. On the other hand, the lowest energy state has quite low absorption cross section leading to a weak signal in $2 \mathrm{D}$ electronic spectra, which could be affected by the ESA.

The significantly broader lineshape of the states at wavelengths shorter than $670 \mathrm{~nm}\left(100-200 \mathrm{~cm}^{-1}\right)$ is an indication of the existence of additional exciton levels in this region in the real system, which is omitted in the model. Therefore, the spectral and kinetic information in the short-wavelength region is less detailed and interpretation of the EET pathways obtained by the fit needs to reflect this.

In our model, the homogeneous linewidths of the exciton states are independent unconstrained fit parameters describing the $2 \mathrm{D}$ peakshapes of the respective diagonal peaks. Figure 3 presents the distributions of the homogeneous linewidths (HWHM) of the eight excitons. For the six lowest-energy states [Figs. 3(c)-3(h)], the homogeneous linewidth is roughly within $20-35 \mathrm{~cm}^{-1}$. The $673 \mathrm{~nm}$ state [Fig. 3(e)] is slightly narrower than others $\left(16-22 \mathrm{~cm}^{-1}\right)$. The two higher-energy states at $655 \mathrm{~nm}$ [Fig. 3(a)] and $664 \mathrm{~nm}$ [Fig. 3(b)] are broad due to the high uncertainty in fitted results as
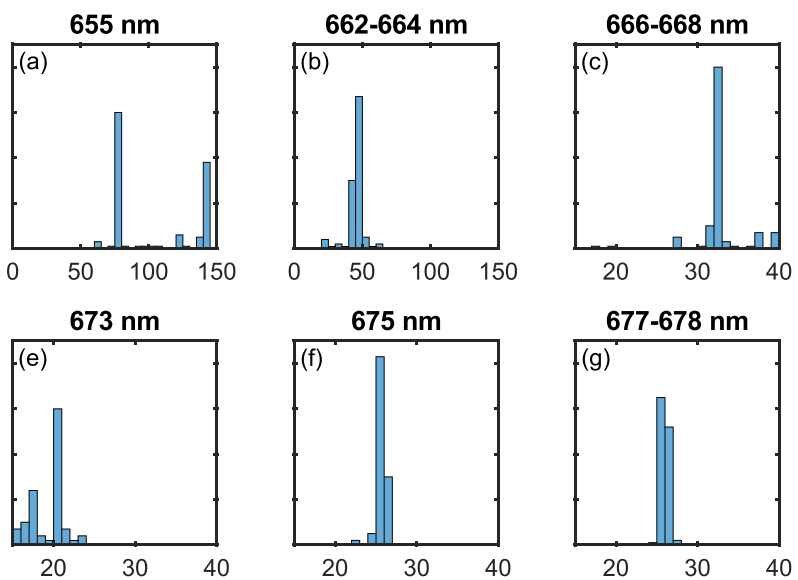

$670-671 \mathrm{~nm}$

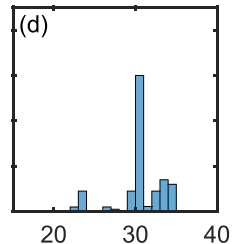

680-681 $\mathrm{nm}$

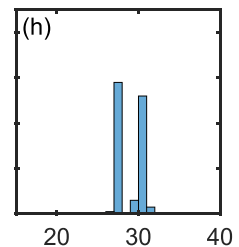

FIG. 3. [(a)-(h)] Homogeneous linewidth (antidiagonal HWHM of the 2D peakshapes) distributions of the eight excitonic levels obtained from the best 120 fits. The bold title of each panel presents the wavelength of the corresponding excitonic level, and the horizontal axis shows the linewidth in unit of $\mathrm{cm}^{-1}$. 
discussed above. Compared with the reported homogeneous linewidth (HWHM) of Chl $a$ in LHCII of $<1.5 \mathrm{~cm}^{-1}$ obtained by single molecule spectroscopy at $1.8 \mathrm{~K}^{34}$ and $65 \mathrm{~cm}^{-1}$ obtained from $2 \mathrm{DES}$ measurement at room temperature, ${ }^{20}$ we can interpolate to estimate the homogeneous linewidths at $77 \mathrm{~K}$ around $18 \mathrm{~cm}^{-1}$, which is in the range of our results. Thus, the modeling approach reliably retrieves the homogeneous linewidths for, at least, the lowest six excitons.

\section{B. Variation in the fitted kinetic parameters}

The dynamics of exciton relaxation is described by the transferrate matrix $\mathbf{K}$. To investigate the robustness of the model, we compare the transfer rate constants obtained from the 120 best fits (Fig. 4). Because of the large number of fit parameters and limited spectral resolution and dynamic range, the optimization can reach multiple solutions having equivalent goodness-of-fit (within numerical noise). However, the discrete distributions of $\mathbf{K}$ elements show that, in practice, only a small number of different solutions exist (within the given eight-exciton parameter space). Moreover, all solutions share common characteristics, for example, the fit parameters (both spectral and dynamic) of the lowest three excitons are very similar. We conducted k-means clustering analysis applying the Lloyd algorithm ${ }^{35}$ to group the 120 best-fit $\mathbf{K}$ matrices into the clusters of similar solutions. As a result of the kmeans analysis, the rate constants in Fig. 4 are grouped into two clusters (indicated by red and blue colors). We note that if we increase the number of excitons into 9 states, the k-means clustering analysis cannot be performed successfully due to the very high heterogeneity of the obtained solutions. Therefore, within our signal-to-noise ratio, an 8 -exciton model is the most reasonable choice.

One cluster, indicated in blue, is highly homogeneous, that is, the rate constants (as well as the rest of the model parameters) have virtually identical values among all solutions. The second cluster (red) also exhibits high degree of overall homogeneity except for some elements of the $\mathbf{K}$ matrix, which vary among solutions. The variability is most pronounced for the $655 \mathrm{~nm}$ and $664 \mathrm{~nm}$ excitons (the two left columns in Fig. 4) and, to some extent, for the $668 \mathrm{~nm}$ exciton, whereas the differences among the rest of the $\mathbf{K}$ matrix elements are largely inconsequential. Thus, there is ambiguity in the model regarding the high-energy excitons, which can be understood considering the lower excitation intensity in this range combined with the fact that the states are relatively short-lived. Overall, we find that the randomized serial optimization routine returns two distinct clusters of highly homogeneous solutions. Furthermore, we select representative solutions from the red and blue clusters and refer to them as "model A" and "model B", respectively. We next compare

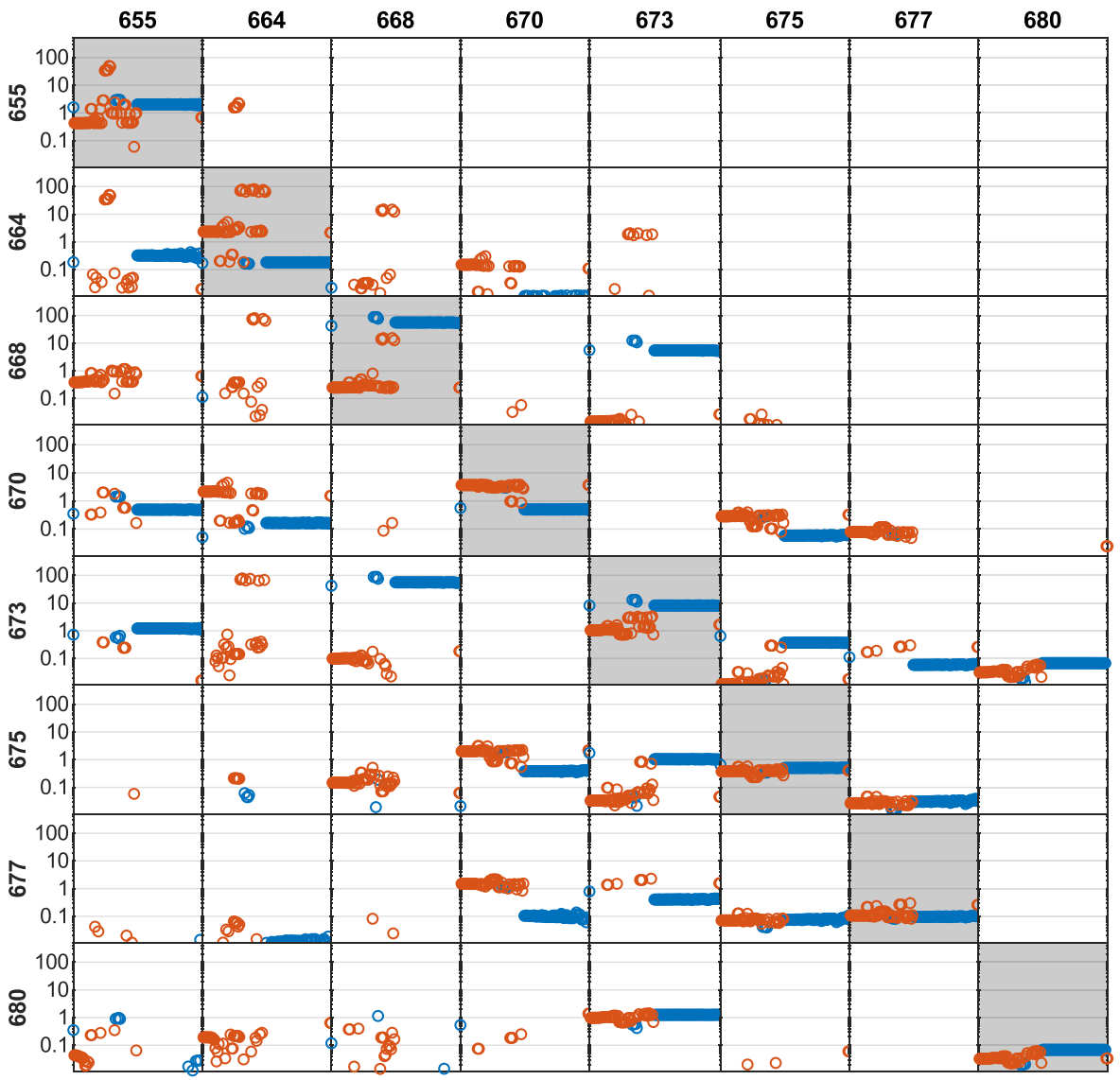

FIG. 4. Transfer-rate matrix $\mathbf{K}$ grid showing the transfer rate constant values of the 120 lowest-residual fits. The vertical axis shows the EET rate values in unit of $\mathrm{ps}^{-1}$. The data points are plotted in a sorted order of increasing mean squared error (from left to right). The residual variation within the best 120 fits is negligible $(<1 / 1000$ compared to the highest residual fit). Rows and columns in the grid represent the donor and acceptor states, respectively, and the diagonal cells (shaded) show the total decay rates of the corresponding excitonic levels. The values are grouped in two clusters, indicated by the symbol color, based on $k$-means clustering analysis. 

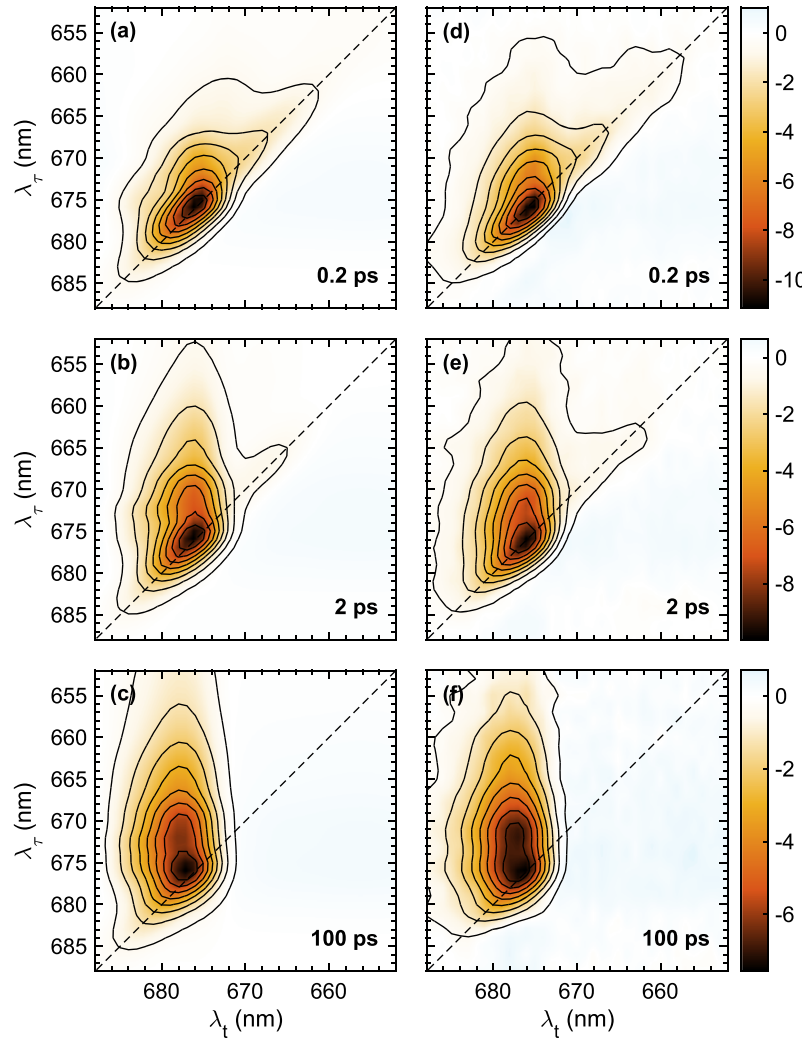

FIG. 5. Comparison between the simulated $2 \mathrm{D}$ electronic spectra obtained from the representative fit of the model $A[(a)-(c)]$ vs the corresponding experimental ones [(d)-(f)] at waiting times of $T_{w}=0.2,2$, and $100 \mathrm{ps}$.

and contrast the fitting results from models $\mathrm{A}$ and $\mathrm{B}$ together with the experimental data with the aim to extract the major dynamics information in LHCII.

\section{Simulated 2D electronic spectra and spectral evolution kinetics}

Figure 5 compares 2D electronic spectra calculated using model A [(a)-(c)] and the corresponding experimental ones [(d)-(f)] at selected $T_{w} \mathrm{~s}$. The fit qualitatively reproduced the main spectral features of the experimental spectra. In the experimental 2D spectrum at early waiting time $T_{w}=200 \mathrm{fs}$, the main spectral features concentrate at the lower energy region $\left(\lambda_{\tau}>670 \mathrm{~nm}\right)$, and within this region, the simulated spectrum replicates the experimental result very well. The simulated spectrum differs from the experimental one mainly in the region $\lambda_{\tau}<660 \mathrm{~nm}$, underestimating diagonal and off-diagonal, $\left(\lambda_{\tau}, \lambda_{t}\right)=(660,676 \mathrm{~nm})$, signals visible in the experimental spectrum. This deviation is an indication that the model lacks some states absorbing in this short-wavelength region (below $660 \mathrm{~nm}$ ), evidently because the weak intensity signals (due to the lower excitation intensity) have less statistical weight.

The $2 \mathrm{D}$ electronic spectrum at $T_{w}=2$ ps is very well reproduced by the fit. The cross-peak signal at $\left(\lambda_{\tau}, \lambda_{t}\right)=(660,676 \mathrm{~nm})$ is comparable between the fit and the experimental spectra. The elongated diagonal feature at $662-670 \mathrm{~nm}$ in the experimental spectrum is still slightly underestimated in the fit, further suggesting that there are one or more additional excitonic states in the real system within this spectral region. The diagonal signal is discerned clearly after 4-5 ps in agreement with the existence of a long-lived "bottleneck" state in this wavelength region revealed in numerous earlier studies. ${ }^{16,17,25}$ Another difference between the fit and the experimental spectra at $T_{w}=2 \mathrm{ps}$ is the absence of the off-diagonal feature around $\left(\lambda_{\tau}, \lambda_{t}\right)=(674,680 \mathrm{~nm})$. This missing feature causes a "kink" in the contour lines of the fitted $2 \mathrm{D}$ spectrum which is not visible in the experimental one.

At $T_{w}=50$ ps and longer, EET has virtually completed, which is evidenced by the invariant shape of the $2 \mathrm{D}$ electronic spectra (not shown, but similar to the 100-ps 2D spectrum). All spectral features are vertically aligned at the emission wavelength of $680 \mathrm{~nm}$, and the fit can reproduce this feature very well. The diagonal peaks in the $675-680 \mathrm{~nm}$ region remain elongated along the diagonal, which is apparent from the asymmetric spacing of the contour lines around this region. This spectral feature is reproduced by the model by way of an inhomogeneously broadened lineshape of the lowest-energy states. At $77 \mathrm{~K}$, the protein can be frozen in multiple conformations, resulting in a static energetic disorder, i.e., variation in the absorption frequencies, manifested as inhomogeneously broadened $2 \mathrm{D}$ peaks.

The fitting quality of model B is very similar, and its comparison with the experimental spectra is presented in Fig. S2 of the supplementary material.

Next, we compare the fitting results of the kinetic traces between the two models. The kinetic traces are more important as they are directly connected to the EET network. The analysis of the EET kinetics is based on a comparison of the model and experimental time courses at different excitation/detection wavelengths. Figure 6 presents the fitting results for representative kinetic traces obtained from model A (continuous curves) and model B (dashed curves) for selected diagonal [Fig. 6(a)] and off-diagonal [Fig. 6(b)] wavelengths. The simulated traces capture the main kinetic features, especially the traces of diagonal peaks in the range of 664-674 nm. For the $674 \mathrm{~nm}$ diagonal trace, the modeled signal intensity at $T_{w}<1 \mathrm{ps}$ is slightly underestimated by both models. The decay of the long-wavelength (in the range of $674-678 \mathrm{~nm}$ ) diagonal signals at $T_{w}>30$ ps is represented better by model $\mathrm{B}$, whereas model A produces traces that overestimate the decay, with very slow decay lifetimes ( $>100 \mathrm{ps})$, which will be discussed later.

The cross-peak dynamics is also reproduced quite well within the experimental noise level, especially between the time of $T_{w}=0.5-20$ ps. At very short times, some cross-peak amplitudes are underestimated in the models [e.g., blue and red traces in Fig. 6(b)]. The deviation indicates that the models omit some very fast relaxation processes or cross-peaks at zero waiting times, or both. Additionally, model B deviates from the experimental dynamics at $\left(\lambda_{\tau}, \lambda_{t}\right)$ $=(673,675 \mathrm{~nm})$, colored in yellow, in the $1-2$ ps time range. On the other hand, as mentioned above, model B describes better dynamics at $T_{w}>30$ ps. Therefore, from the rough comparison, we can recognize the trade-off in models $\mathrm{A}$ and $\mathrm{B}$, which result in better representation of the dynamics at shorter and longer time range, respectively. 

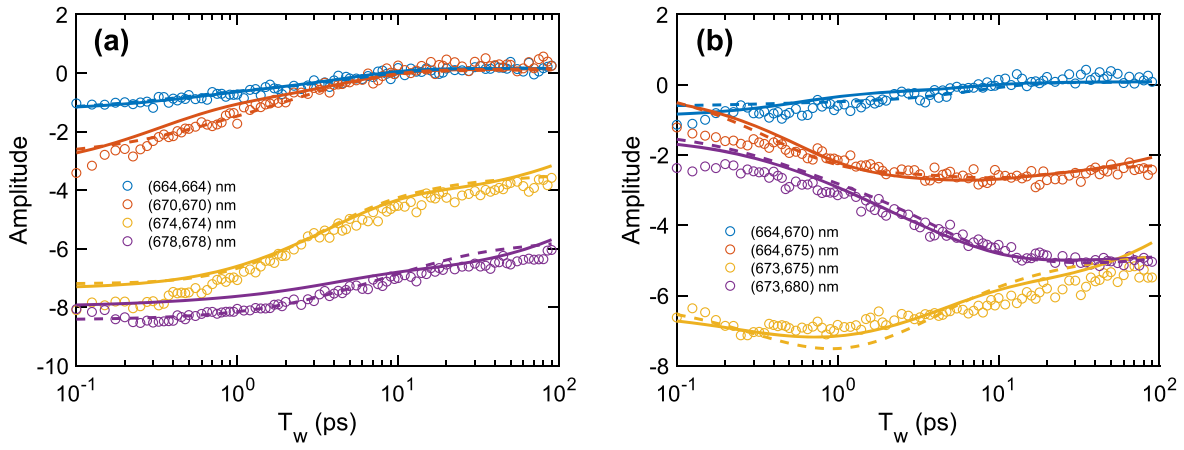

FIG. 6. Representative kinetic traces for selected diagonal peaks (a) and for cross-peaks (b). The legend indicates the coordinates of the peaks as $\left(\lambda_{\tau}, \lambda_{t}\right) \mathrm{nm}$. The model $A$ and model $B$ fit results are shown as continuous curves and dashed curves, respectively, and the symbols are the experimental data.

\section{Exciton state connectivity and dynamics}

The kinetics of the system is described by two matrices-the $\mathbf{X}$ matrix of zero-time cross-peak amplitudes and the transfer-rate matrix $\mathbf{K}$. The best-fit values of $\mathbf{K}$ obtained from model A and model $\mathrm{B}$ are presented in Figs. 7(a) and 7(b), respectively, with the shading colors indicating the corresponding elements of the $\mathbf{X}$ matrix. Due to the limited experimental time resolution, the fast EET processes occurring between strongly coupled pigments in the time scale of tens of femtoseconds are not resolved in the 2DES data. ${ }^{16}$ These processes are partially compensated by the $\mathbf{X}$ matrix, which essentially provides instantaneous redistribution of the excitation energy between the strongly coupled states. Hence, the transfer-rate matrix $\mathbf{K}$ alone does not provide a comprehensive picture of EET network and must be analyzed concurrently with the $\mathbf{X}$ matrix to provide a physically reasonable EET scheme. The decay lifetimes of the system, which are obtained as the reciprocal eigenvalues of the transferrate matrix, and the corresponding population amplitudes (weighted eigenvectors) are reported in Fig. 8. Finally, Fig. 9 shows the simulated population dynamics of the eight excitons after uniform initial excitation.

\section{Strongly coupled exciton states}

Inspection of the $\mathbf{X}$ matrix gives hints about the strongly coupled states, which result in significant cross-peak intensity at early waiting times. It is important to note that the $\mathbf{X}$ matrix elements are constrained within \pm 0.5 . The values of 0.5 , represented by the orange shading in Fig. 7 , indicate a strong coupling and relatively fast $(<100 \mathrm{fs})$ relaxation, between two excitons. Strong coupling is also described by a large transfer rate constant $\left(>10 \mathrm{ps}^{-1}\right)$. Both models $\mathrm{A}$ and $\mathrm{B}$ contain high-valued off-diagonal $\mathbf{X}$ elements between

\begin{tabular}{|r|r|r|r|r|r|r|r|r|}
\hline (a) & 655.0 & 664.0 & 668.0 & 670.3 & 672.6 & 675.1 & 677.5 & 681.0 \\
\hline 655.0 & $\mathbf{- 0 . 4 3}$ & & & & & & & \\
\hline 664.0 & & $\mathbf{- 2 . 3}$ & & 0.15 & & & & \\
\hline 668.0 & 0.39 & & $\mathbf{- 0 . 2 5}$ & & 0.01 & 0.01 & & \\
\hline 670.3 & & 2.1 & & $\mathbf{- 3 . 7}$ & & 0.28 & 0.08 & \\
\hline 672.6 & & & 0.10 & & $\mathbf{- 1 . 0 3}$ & 0.01 & & 0.03 \\
\hline 675.1 & & & 0.15 & 2.0 & 0.03 & $\mathbf{- 0 . 3 7}$ & 0.03 & \\
\hline 677.5 & & & & 1.5 & & 0.07 & $\mathbf{- 0 . 1 1}$ & \\
\hline 681.0 & 0.04 & 0.19 & & & 0.98 & & & $\mathbf{- 0 . 0 3}$ \\
\hline
\end{tabular}

\begin{tabular}{|r|r|r|r|r|r|r|r|r|}
\hline (b) & 655.0 & 664.0 & 666.9 & 670.4 & 672.5 & 675.0 & 677.1 & 679.6 \\
\hline 655.0 & $\mathbf{- 2 . 0}$ & 0.01 & & & & & & \\
\hline 664.0 & 0.32 & $\mathbf{- 0 . 1 8}$ & & 0.01 & & & & \\
\hline 666.9 & & & $\mathbf{- 5 6}$ & & 5.5 & & & \\
\hline 670.4 & 0.49 & 0.16 & & $\mathbf{- 0 . 4 9}$ & & 0.06 & 0.01 & \\
\hline 672.5 & 1.2 & & 56 & & $\mathbf{- 8 . 2}$ & 0.37 & 0.06 & 0.07 \\
\hline 675.0 & & & & 0.38 & 1.0 & $\mathbf{- 0 . 5 0}$ & 0.03 & \\
\hline 677.1 & & 0.01 & & 0.10 & 0.40 & 0.07 & $\mathbf{- 0 . 1 0}$ & \\
\hline 679.6 & & & & & 1.3 & & & $\mathbf{- 0 . 0 7}$ \\
\hline
\end{tabular}

FIG. 7. Fitting result for the transfer-rate matrix $\mathbf{K}$ obtained from (a) model $A$ and (b) model B. The entries are arranged similarly to Fig. 4. The orange and yellow shading cells indicate strong and weak couplings reflected by $\mathbf{X}$ matrix elements.

\begin{tabular}{|r|r|r|r|r|r|r|r|r|}
\cline { 2 - 8 } (a) & 0.2 & 0.5 & 0.9 & 2.3 & 3.4 & 4.4 & 170 & 2000 \\
\hline 655.0 & 0.00 & 0.00 & 0.00 & 0.97 & 0.01 & 0.02 & 0.00 & 0.00 \\
\hline 664.0 & 0.01 & 1.47 & 0.00 & 0.01 & -0.03 & 0.02 & 0.01 & 0.01 \\
668.0 & 0.00 & 0.01 & -0.04 & -2.07 & 0.87 & 2.64 & 0.03 & 0.05 \\
\hline 670.3 & -0.06 & 1.68 & -0.01 & 0.12 & -0.35 & 0.31 & 0.17 & 0.14 \\
\hline 672.6 & 0.00 & 0.02 & 2.41 & -0.28 & 0.03 & 0.34 & -0.13 & 0.35 \\
\hline 675.1 & 0.04 & -1.88 & -0.08 & 1.57 & -5.36 & 5.62 & 1.19 & 1.00 \\
\hline 677.5 & 0.02 & -1.16 & 0.02 & -0.90 & 4.92 & -7.21 & 3.40 & 2.66 \\
\hline 681.0 & 0.00 & -0.14 & -2.30 & 0.58 & -0.09 & -1.74 & -4.68 & 11.10 \\
\hline
\end{tabular}

\begin{tabular}{|r|r|r|r|r|r|r|r|r|}
\cline { 2 - 8 }$(\mathrm{b})$ & 0.016 & 0.4 & 0.5 & 1.7 & 3.3 & 5.7 & 13 & 2000 \\
\hline 655.0 & 0.00 & 0.00 & 0.99 & 0.00 & 0.00 & 0.01 & 0.00 & 0.00 \\
\hline 664.0 & 0.00 & 0.00 & -0.17 & -0.01 & -0.02 & 1.49 & 0.01 & 0.01 \\
\hline 666.9 & 0.72 & 0.06 & 0.16 & -0.01 & 0.01 & 0.01 & 0.00 & 0.04 \\
\hline 670.4 & 0.00 & 0.01 & -0.26 & 0.25 & 0.27 & 0.91 & 0.09 & 0.19 \\
\hline 672.5 & -0.76 & 0.59 & 1.56 & -0.06 & 0.13 & 0.07 & 0.03 & 0.45 \\
\hline 675.0 & 0.01 & -0.29 & -1.00 & -0.38 & 1.05 & 1.01 & 0.33 & 1.26 \\
677.2 & 0.00 & -0.09 & -0.27 & 0.06 & -0.76 & -2.69 & 2.59 & 3.11 \\
\hline 679.6 & 0.02 & -0.29 & -1.01 & 0.15 & -0.68 & -0.79 & -3.04 & 8.36 \\
\hline
\end{tabular}

FIG. 8. Population amplitudes (weighted eigenvectors) table of the transfer-rate matrix K obtained from (a) model A and (b) model B. The first row represents the dynamics lifetimes in unit of picosecond calculated from the reciprocal of eigenvalue of the corresponding transfer-rate matrix $\mathbf{K}$. The first column is the fit results of excitonic level in $\mathrm{nm}$. 

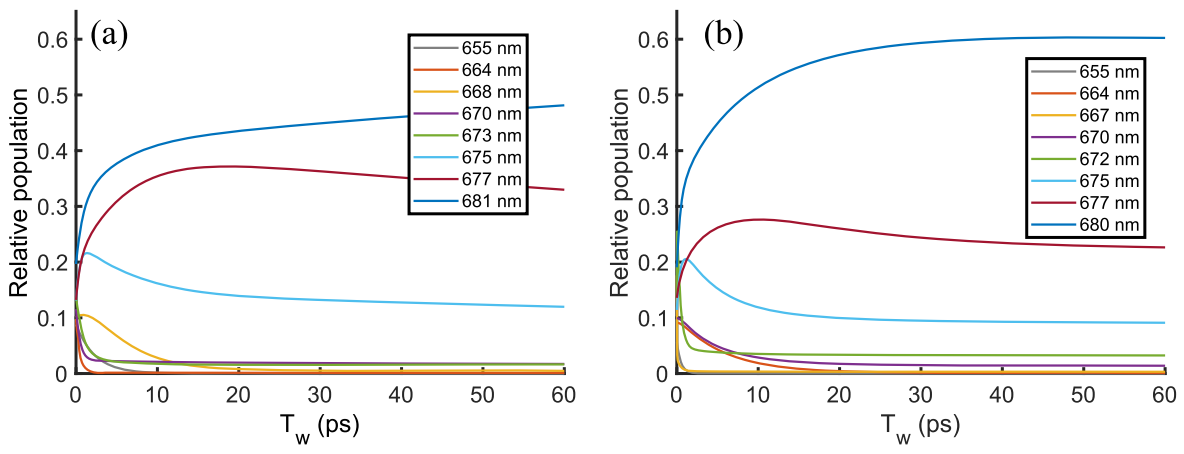

FIG. 9. The simulation of population dynamics of eight excitons obtained from (a) model A and (b) model B, and the initial excitation density is uniform amongst all eight states

664-667 $\mathrm{nm}$ and 670-673 $\mathrm{nm}$ and between $667-670 \mathrm{~nm}$ and $677-$ $680 \mathrm{~nm}$. In model $\mathrm{A}$, these connections are entirely within the $\mathbf{X}$ matrix, whereas in model $B$, there is a fast relaxation between $667 \mathrm{~nm}$ and $673 \mathrm{~nm}$. Note that the exact time scale is not reliably determined as the rate constant of $56 \mathrm{ps}^{-1}$ is beyond the experimental time resolution. The rapidly relaxing states at $664-667$ and $667-670 \mathrm{~nm}$ at least partly represent higher exciton states in the strongly coupled Chl $a$ domains.

Several other exciton states relax on time scales $0.1-1$ ps. In model $\mathrm{A}$, these are at $664 \mathrm{~nm}(0.5 \mathrm{ps}$ transfer time to $670 \mathrm{~nm})$, $670 \mathrm{~nm}(0.3-$ ps transfer to $675-677 \mathrm{~nm})$, and $673 \mathrm{~nm}(1 \mathrm{ps}-680 \mathrm{~nm})$. In model $\mathrm{B}$, we observe rapid transfer from $673 \mathrm{~nm}$ to $675-679 \mathrm{~nm}$. Interestingly, a rate constant of $\sim 1 \mathrm{ps}^{-1}$ between 673 and $680 \mathrm{~nm}$ is consistently determined in all 120 best fits (see Fig. 4).

\section{The "bottleneck" intermediate state}

Both models A and B predict at least one long-living state absorbing between $664 \mathrm{~nm}$ and $668 \mathrm{~nm}$, which is weakly connected to other Chls and evidently represents the so-called "bottleneck" state, identified in numerous earlier studies. ${ }^{15-17,23-25}$ The total depopulation rate constant is $0.18-0.25 \mathrm{ps}^{-1}$. Note that this value is highly consistent among all fits in the respective $\mathrm{A}$ and $\mathrm{B}$ clusters. The corresponding decay lifetime of the long-lived state is 4.4 ps in model A, indicated in Fig. 8(a) by the high positive contribution of the $668-\mathrm{nm}$ state to the weighted eigenvector of 4.4-ps lifetime. In model B, the lifetime of this long-lived state is predicted as $5.7 \mathrm{ps}$, indicated in Fig. 8(b) by the relatively high contribution of the 664-nm exciton to the weighted eigenvector of 5.4-ps lifetime. A second long-lived state at $670 \mathrm{~nm}$ is predicted in model $\mathrm{B}$, which is the main contributor to the $1.7 \mathrm{ps}$ lifetime. In the alternative model $\mathrm{A}$, the $670 \mathrm{~nm}$ decays mainly with a 0.5 -ps lifetime. It must be said that both dynamic components can be observed in the experimental 2D decay-associated spectra (DAS) (Fig. S2 of the supplementary material), whereas the model is restricted to a single state at $670 \mathrm{~nm}$. Similarly, the long-lived state in both models appears strongly coupled to lower excitons via the $\mathbf{X}$ matrix so that $50 \%-60 \%$ of the population is instantaneously redistributed. This can be explained if the excitonic level at $664 \mathrm{~nm}(668 \mathrm{~nm}$ in model B) represents two degenerate states, one rapidly and the other slowly relaxing.

Earlier experimental results suggested that the $664 \mathrm{~nm}$ state also contributes significantly to the $12-15$ ps decay lifetime. ${ }^{6,36}$
However, the present modeling clearly separates the decay of the $664 \mathrm{~nm}$ state occurring on a $4-6$ ps time scale from the $12-15 \mathrm{ps}$ decay lifetime, which reflects equilibration among the energy sinks (see below)

\section{Energy transfer between the lowest-energy sinks}

All fit results are highly uniform with respect to the lowest three exciton states, 675, 677-678, and 680-681 nm, which act as final energy sinks populated from higher exciton levels mostly within 1 ps (Fig. 9) and slowly equilibrating thereafter. A striking result is the lack of significant direct EET among these three sinks. The result is roughly in line with the calculations of Renger et al. ${ }^{17}$ who predict rather slow exchanges between the energy sinks. Nevertheless, the excitation energy is slowly equilibrated. This is possible because even at $77 \mathrm{~K}$, there is non-negligible repopulation of higher-energy exciton levels in the strongly coupled Chl $a$ pools. The higher exciton levels effectively bridge the different pools so that an equilibrium is established irrespectively of the initial excitation. This is demonstrated by the population kinetics with different initial excitations in Fig. S4 of the supplementary material. In this scenario, energetically uphill pathways are critical for spatial EET within the complex, and their enhancement at higher, physiologically relevant temperature will result in overall acceleration of EET, which has been observed experimentally. ${ }^{22}$ Bridging pools of low-energy states via uphill transfer to intermediate states is a crucial design principle for photosynthetic complexes, and especially supercomplexes, to ensure successful transfer of excitations irrespective of the photon energy and to avoid excitation trapping.

The $675-\mathrm{nm}$ state equilibrates faster (via $670-673-\mathrm{nm}$ states), on a time scale of 3-6 ps. The energy transfer from the 677-678-nm state is slower, and the two fit clusters diverge in the best fit lifetime -170 ps for model A and 13 ps for model B. However, as it was mentioned earlier, model A deviates from the experimental kinetics beyond 50 ps, while the 13-ps 2D DAS simulated by model $\mathrm{B}$ is qualitatively similar to the experimental 15-ps 2D DAS (Fig. S2 of the supplementary material).

The final state, which is reached in approximately 40 ps in model B [Fig. 9(b)], is an equilibrium mainly among the lowest three excitons, with $60 \%$ population of the $680-681-\mathrm{nm}$ exciton. This is consistent with the finding that at $77 \mathrm{~K}$, there are multiple fluorescence emitting states in LHCII. ${ }^{37-39}$ 


\section{E. Assignment of exciton states to chlorophylls in LHCII}

The phenomenological model described here provides a great deal of insight into the dynamics of EET without requiring substantial a priori knowledge of the system. However, it does not directly link spectral features with molecular entities (Chls), which is necessary to translate the spectral evolution into the spatial energy transfer. A microscopic model of EET can be proposed (Fig. 10) considering the available knowledge of the Chl site energies and exciton couplings, obtained from mutagenesis studies, structurebased quantum modeling and fitting to steady-state, and timeresolved spectroscopy data. ${ }^{14-17,40}$ We must stress, however, that the model is only tentative and serves mainly to analyze the experimental dynamics in the framework of detailed structure-based exciton calculations. To create the scheme, we make the following assumptions and considerations:

- The highest exciton states at $655 \mathrm{~nm}$ and $664 \mathrm{~nm}$ are assigned to Chl $b$ and all lower states to Chl $a$.

- The 668-nm state (664-nm in model B) is long-lived, decaying in 4-6 ps via slow branched channels to the main Chl $a$ groups. This state can be assigned to Chl $a 604$, according to Novoderezhkin, ${ }^{16}$ but it could also be contributed by a red-shifted Chl $b$.

- Exciton states in the model, including the 668-nm state, which are weakly coupled to other states via the transferrate matrix $\mathbf{K}$ (with a total depopulation time of $>0.5 \mathrm{ps}$ ) but are strongly coupled via off-diagonal $\mathbf{X}$ matrix elements are considered as representing two degenerate excitons.

- Because of the slow energy exchange between the three lowest exciton states, 675, 677-678, and 680-681-nm states, they are interpreted as each belonging to one of the different Chl a exciton domains, encompassing Chls $a 602 / 603, a 610 / 611 / 612, a 613 / 614$, and possibly Chl $a 604$.

- The lowest-energy state (680-681 nm) and the higher levels strongly coupled to it via the $\mathbf{X}$ matrix (at $668 \mathrm{~nm}$ and $673-$ $678 \mathrm{~nm}$ ) belong to the Chl $a 610 / 611 / 612$ group. ${ }^{15-17}$ The relaxation from the higher exciton levels is not resolved in the transfer-rate matrix $\mathbf{K}$, indicating that it occurs mostly under $100 \mathrm{fs}$.

- The states at $675 \mathrm{~nm}$ and $677-678 \mathrm{~nm}$ are then tentatively ascribed to the $a 602 / 603$ and $a 613 / 614$ groups. The $0.4-$ 0.5 ps decay from $670 \mathrm{~nm}$ could be attributed to relaxation within the 670-675-nm exciton dimer.

- The 673-nm level relaxes (1 ps) to the lowest-energy state but is also connected to the $675-\mathrm{nm}$ exciton via $\mathbf{X}$. In model B, the relaxation is branched to all lower states. We can interpret this state as the upper level of the 673-678-nm exciton dimer. In this case, by comparing with previous theoretical predictions, we identify the dimer as the $a 602 / 603$ pair, in which ps relaxation can be expected due to the relatively weak coupling. ${ }^{16,17,41}$ Alternatively, if the site energy calculation by Renger et al. ${ }^{17}$ is considered, this state can also be attributed to Chl a604.

- Equilibration between the $675 \mathrm{~nm}$ and the 677-678 nm pools occurs on a time scale of 2-6 ps. The slowest dynamics involves equilibration between the 677-678-nm and the $680-681-\mathrm{nm}$ pools, occurring on a time scale of $10-15$ ps. The latter is consistent with EET between the stromal and lumenal pigment groups in Novoderezhkin's model.

Without a definitive knowledge of the site energies and exciton couplings of all Chls in LHCII, only speculative assignments can be made. Furthermore, the connectivity between the exciton states is
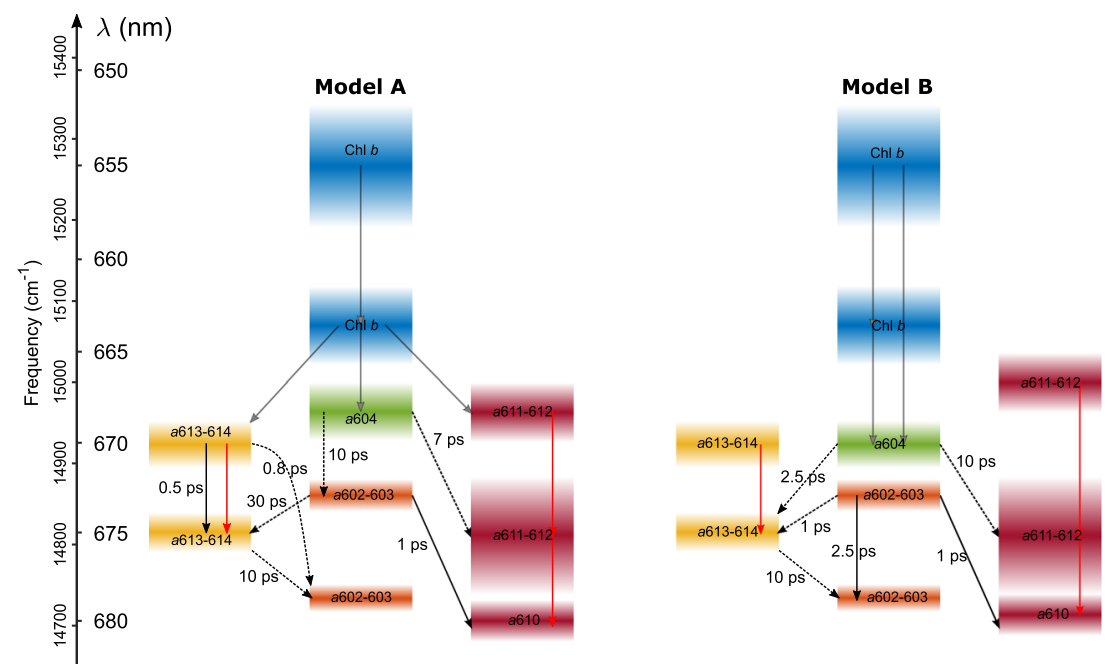

FIG. 10. EET schemes in trimeric LHCII at $77 \mathrm{~K}$ constructed based on fitting results in model A and model $\mathrm{B}$. The red arrows indicate the fast exciton relaxation within the strongly coupled domains. The black dash arrows indicate transmembrane EET happening between pigments located on two different membrane sides. Black line arrows indicate the EET happening within the same membrane side. The gray arrows indicate the fast EET happening within Chls $b$ manifold or from Chls $b$ to Chls a. Only the downhill transfers are presented. The colors of excitonic levels indicate strongly coupled domains, except the blue color is used to represent the $\mathrm{Chl} b$ manifold. The pigment assignments are speculative. See the main text for more details on the interpretations. 
not fully determined by the modeling due to the limited time and spectral resolution of the data. It must be stressed that because of structural heterogeneity (static and dynamic), the real system cannot be fully understood by a one-to-one mapping of absorption bands to individual Chl exciton states, neither can a single matrix of microscopic rate constants fully describe the dynamics. Small conformational changes in LHCII can result in variations in site energies and in exciton couplings that substantially alter the energy landscape and dynamics, which are not resolved in the ensemble-averaged spectroscopy data.

\section{CONCLUSIONS}

In this study, we construct a phenomenological model describing the EET process in trimeric LHCII at $77 \mathrm{~K}$. We demonstrate that by simultaneously fitting the spectral and kinetic model parameters to the 2DES data along with a robust sampling of the initial parameter space and clustering the fitting results, we can resolve greater details in the spectral composition and the excitation kinetics. In this way, we were able to determine the position of five excitonic levels, at $670-671,673,675,677-678$, and 680-681 nm, with very high accuracy. Not only the central wavelengths but also the inhomogeneous and homogeneous linewidths are consistently determined amongst the five lowest states in the range of $30-55 \mathrm{~cm}^{-1}$ and $20-35 \mathrm{~cm}^{-1}$, respectively. In this way, the $2 \mathrm{DES}$ data fitting is a practical approach to measure the homogeneous linewidth, which is not accessible by conventional TA spectroscopy. The positions and widths for the higher excitonic states are not precisely defined here but can be determined, in principle, by this approach, provided that higher signal-to-noise data are available in this region. For future studies, where a broadband excitation can be deployed to cover the whole Chl $a$ and Chl $b$ Qy bands, the vibronic bands of the Chls should be taken into consideration. In this work, as most of the vibronic bands of Chls $a$ are located out of our excitation spectral bandwidth, we have only modeled the lineshape of each exciton level by a Gaussian function.

Within the limits of time and spectral resolution and the signalto-noise level in the data along with the high dimensionality of the model, it is inevitable that there is no unique solution with respect to the connectivity and dynamics of the system. Clustering analysis classified the fitting results into two groups of alike solutions (A and B). Within one cluster, variations were observed mostly within the highest three exciton states, where the signal-to-noise level is relatively low and different permutations of kinetic parameters result in a similar goodness-of-fit. Comparatively, analyzing the two alternative models, we found that model A generally describes better the dynamics at early waiting times and model B performs better for the slower dynamics. Either model omits some details in the kinetics, which is a necessary compromise set by the restriction of eight exciton components. Another limitation of the presently available data is that the dynamics in the first $100 \mathrm{fs}$ and the excitonic states relaxing within this period are not resolved.

Despite these limitations, the phenomenological modeling converges to a reasonable exciton relaxation scheme, which in many aspects agrees with other models constructed by structure-based calculations $^{16,17}$ and time-resolved data. ${ }^{23,25}$ Although a long-lived state absorbing in the region $664-668 \mathrm{~nm}$ is positively identified by the modeling, its lifetime of $4-6$ ps is markedly shorter than earlier predictions. The modeling identifies three energy sinks in LHCII, at $675,677-678$, and $680-681 \mathrm{~nm}$ with no significant EET between them. Equilibration between those three $\mathrm{Chl} a$ pools occurs on time scales from a few picoseconds to tens of picoseconds via upperlying exciton states and will therefore exhibit pronounced temperature dependence, which is partly the reason for the faster EET in LHCII observed at room temperature. ${ }^{22}$ The final state is a thermal equilibrium among primarily the three sinks, with the lowest state $(680-681 \mathrm{~nm})$ having approximately $60 \%$ population. Finally, according to the model, this equilibrium is independent of the initial excitation conditions, which is reasonable in the design perspective of the light-harvesting antennas and supercomplexes to ensure the photon-capturing function under different light conditions. However, due to static energy disorder (inhomogeneous broadening), the $2 \mathrm{D}$ electronic spectra at $77 \mathrm{~K}$ still exhibits a certain degree of correlation between excitation and detection wavelength in the long-wavelength region.

Although the phenomenological modeling is capable of extracting more details from the experimental 2DES data, than, e.g., global lifetime analysis, ultimately structure-based quantum modeling together with data fitting is necessary to understand the photophysics of the complex in molecular terms. However, the approach described here can also be applied in the future for different lightharvesting antenna complexes, whose atomic structural information is not available.

\section{SUPPLEMENTARY MATERIAL}

See the supplementary material for more details on (I) 2D peakshape, (II) phenomenological ESA spectrum, (III) eigenstate comparison, (IV) global analysis, (V) energy equilibration within the lowest excitonic levels, (VI) quantum beats, and (VII) number of states.

\section{ACKNOWLEDGMENTS}

The authors acknowledge support from the Hungarian Ministry of Innovation and Technology, National Research, Development and Innovation Office (Grant Nos. NN-124904 and 20181.2.1-NKP-2018-00009 to P.H.L.) and the Singapore Ministry of Education, Academic Research Fund (Tier 2 Grant No. MOE2015T2-1-039 and Tier 1 Grant No. RG15/18 to H.-S.T.). The ELIALPS Project (No. GINOP-2.3.6-15-2015-00001) is supported by the European Union and co-financed by the European Regional Development Fund.

The authors declare no conflicts of interest.

\section{REFERENCES}

${ }^{1}$ R. E. Blankenship, Molecular Mechanisms of Photosynthesis (John Wiley and Sons, 2014)

${ }^{2}$ Z. F. Liu, H. C. Yan, K. B. Wang, T. Y. Kuang, J. P. Zhang, L. L. Gui, X. M. An, and W. R. Chang, Nature 428, 287-292 (2004).

${ }^{3}$ H. van Amerongen and R. Croce, in Primary Processes of Photosynthesis, Part 1: Principles and Apparatus, edited by G. Renger (The Royal Society of Chemistry, Cambridge, UK, 2007), Chap. 8, pp. 329-367.

${ }^{4}$ T. Bittner, K.-D. Irrgang, G. Renger, and M. R. Wasielewski, J. Phys. Chem. 98, 11821-11826 (1994).

${ }^{\mathbf{5}}$ T. Bittner, G. P. Wiederrecht, K.-D. Irrgang, G. Renger, and M. R. Wasielewski, Chem. Phys. 194, 311-322 (1995). 
${ }^{6}$ H. M. Visser, F. J. Kleima, I. H. M. van Stokkum, R. van Grondelle, and H. van Amerongen, Chem. Phys. 210, 297-312 (1996).

${ }^{7}$ C. C. Gradinaru, S. Özdemir, D. Gülen, I. H. M. van Stokkum, R. van Grondelle, and H. van Amerongen, Biophys. J. 75, 3064-3077 (1998).

${ }^{8}$ L. O. Pålsson, M. D. Spangfort, V. Gulbinas, and T. Gillbro, FEBS Lett. 339, 134138 (1994).

${ }^{9}$ J. M. Salverda, M. Vengris, B. P. Krueger, G. D. Scholes, A. R. Czarnoleski, V. Novoderezhkin, H. van Amerongen, and R. van Grondelle, Biophys. J. 84, 450-465 (2003).

${ }^{10}$ J. P. Connelly, M. G. Müller, M. Hucke, G. Gatzen, C. W. Mullineaux, A. V. Ruban, P. Horton, and A. R. Holzwarth, J. Phys. Chem. B 101, 1902-1909 (1997).

${ }^{11}$ R. Agarwal, B. P. Krueger, G. D. Scholes, M. Yang, J. Yom, L. Mets, and G. R. Fleming, J. Phys. Chem. B 104, 2908-2918 (2000).

${ }^{12}$ W. Kühlbrandt, D. N. Wang, and Y. Fujiyoshi, Nature 367, 614-621 (1994).

${ }^{13}$ G. Trinkunas, J. P. Connelly, M. G. Müller, L. Valkunas, and A. R. Holzwarth, J. Phys. Chem. B 101, 7313-7320 (1997).

${ }^{14}$ V. Novoderezhkin, J. M. Salverda, H. van Amerongen, and R. van Grondelle, J. Phys. Chem. B 107, 1893-1912 (2003).

${ }^{15}$ V. I. Novoderezhkin, M. A. Palacios, H. van Amerongen, and R. van Grondelle, J. Phys. Chem. B 109, 10493-10504 (2005).

${ }^{16}$ V. Novoderezhkin, A. Marin, and R. van Grondelle, Phys. Chem. Chem. Phys. 13, 17093-17103 (2011).

${ }^{17}$ T. Renger, M. E. Madjet, A. Knorr, and F. Müh, J. Plant Physiol. 168, 1497-1509 (2011).

${ }^{18}$ P. H. Lambrev, P. Akhtar, and H.-S. Tan, "Insights into the mechanisms and dynamics of energy transfer in plant light-harvesting complexes from twodimensional electronic spectroscopy," Biochim. Biophys. Acta (published online).

${ }^{19}$ K. L. Wells, P. H. Lambrev, Z. Y. Zhang, G. Garab, and H.-S. Tan, Phys. Chem. Chem. Phys. 16, 11640-11646 (2014).

${ }^{20}$ H.-G. Duan, A. L. Stevens, P. Nalbach, M. Thorwart, V. I. Prokhorenko, and R. J. D. Miller, J. Phys. Chem. B 119, 12017-12027 (2015).

${ }^{21}$ M. M. Enriquez, P. Akhtar, C. Zhang, G. Garab, P. H. Lambrev, and H.-S. Tan, J. Chem. Phys. 142, 212432 (2015).

${ }^{22}$ P. Akhtar, C. Zhang, T. N. Do, G. Garab, P. H. Lambrev, and H.-S. Tan, J. Phys. Chem. Lett. 8, 257-263 (2017).
${ }^{23}$ T. R. Calhoun, N. S. Ginsberg, G. S. Schlau-Cohen, Y. C. Cheng, M. Ballottari, R. Bassi, and G. R. Fleming, J. Phys. Chem. B 113, 16291-16295 (2009).

${ }^{24}$ G. S. Schlau-Cohen, T. R. Calhoun, N. S. Ginsberg, M. Ballottari, R. Bassi, and G. R. Fleming, Proc. Natl. Acad. Sci. U. S. A. 107, 13276-13281 (2010).

${ }^{25}$ G. S. Schlau-Cohen, T. R. Calhoun, N. S. Ginsberg, E. L. Read, M. Ballottari, R. Bassi, R. van Grondelle, and G. R. Fleming, J. Phys. Chem. B 113, 15352-15363 (2009).

${ }^{26}$ P. Akhtar, T. N. Do, P. J. Nowakowski, A. Huerta-Viga, M. F. Khyasudeen, P. H. Lambrev, and H.-S. Tan, J. Phys. Chem. B 123, 6765-6775 (2019).

${ }^{27}$ J. Dostál, B. Benešová, and T. Brixner, J. Chem. Phys. 145, 124312 (2016).

${ }^{28}$ E. Thyrhaug, K. Žídek, J. Dostál, D. Bína, and D. Zigmantas, J. Phys. Chem. Lett. 7, 1653-1660 (2016).

${ }^{29}$ D. M. Jonas, Annu. Rev. Phys. Chem. 69, 327-352 (2018).

${ }^{30}$ D. Paleček, P. Edlund, S. Westenhoff, and D. Zigmantas, Sci. Adv. 3, e1603141 (2017).

${ }^{31}$ C. C. Jumper, S. Rafiq, S. Wang, and G. D. Scholes, Curr. Opin. Chem. Biol. 47, 39-46 (2018).

${ }^{32}$ W. T. Pollard and R. A. Friesner, J. Chem. Phys. 100, 5054-5065 (1994).

${ }^{33}$ T. N. Do, M. F. Gelin, and H.-S. Tan, J. Chem. Phys. 147, 144103 (2017).

${ }^{34}$ C. Tietz, F. Jelezko, U. Gerken, S. Schuler, A. Schubert, H. Rogl, and J. Wrachtrup, Biophys. J. 81, 556-562 (2001).

${ }^{35}$ S. Lloyd, IEEE Trans. Inf. Theory 28, 129-137 (1982).

${ }^{36}$ M. A. Palacios, J. Standfuss, M. Vengris, B. F. van Oort, I. H. M. van Stokkum, W. Kühlbrandt, H. van Amerongen, and R. van Grondelle, Photosynth. Res. 88, 269-285 (2006).

${ }^{37}$ J. Pieper, M. Rätsep, K.-D. Irrgang, and A. Freiberg, J. Phys. Chem. B 113, 10870-10880 (2009).

${ }^{38}$ J. Pieper, R. Schödel, K. D. Irrgang, J. Voigt, and G. Renger, J. Phys. Chem. B 105, 7115-7124 (2001).

${ }^{39}$ K. Vrandecic, M. Rätsep, L. Wilk, L. Rusevich, M. Golub, M. Reppert, K.-D. Irrgang, W. Kühlbrandt, and J. Pieper, J. Phys. Chem. B 119, 3920-3930 (2015).

${ }^{40}$ G. Zucchelli, S. Santabarbara, and R. C. Jennings, Biochemistry 51, 2717-2736 (2012).

${ }^{41}$ F. Müh, M. E.-A. Madjet, and T. Renger, J. Phys. Chem. B 114, 13517-13535 (2010). 